\title{
CHARACTERIZATION AND PRODUCTION CONTROL FOR DRIE OF MEMS ACCELEROMETERS USING OPTICAL DISPLACEMENT TRANSDUCER MEASUREMENTS
}

\author{
B. Keese ${ }^{I^{*}}$, B. Homeijer ${ }^{1}$, J.L. Williams ${ }^{1}$ and P. Knutrud ${ }^{2}$ \\ ${ }^{1}$ Hewlett-Packard Co., Technology Development Operations, Corvallis, OR , USA \\ ${ }^{2}$ Inspectrology, Sudbury, MA, USA
}

\begin{abstract}
A Fiber Optic Displacement Transducer is used to measure MEMS device motion after the active elements are released by etching. This paper discusses the use of such measurements to determine the resonant frequency of MEMS devices, the use of this data to characterize and improve Silicon DRIE processing and also describes incorporating this technique as an off-axis measurement system within an existing automated metrology tool. These measurements can be conducted quickly and non-destructively immediately following release etch, and this data has proven more informative than previous methods using the dimensions of etched features to predict the resonant frequency of MEMS devices.
\end{abstract}

\section{INTRODUCTION}

The invention and widespread use of Bosch DRIE processing has facilitated a dramatic increase in the number, type and success of MEMS devices being fabricated [1]. The very deep, high aspect ratio features created using this etch process allow the manufacture of the various sensors, actuators and resonators critical to the MEMS industry. Yet these same dimensional considerations create significant challenges for in-process dimensional measurement. The top-down optical or SEM dimensional measurement traditionally used in microelectronics is often inadequate to characterize and control MEMS processing, and mechanical or beam probes of various sorts are excluded by the geometry of the structures being produced. Thus, an alternate and ultimately more informative measurement has been developed, using a fiber optic lever transducer. These measurements can be conducted quickly and non-destructively immediately following release etch, and the data closely predicts the final performance of the assembled MEMS. This technique has proven more reliable than previous methods which used the dimensions of etched features as a proxy to predict the resonant frequency of the devices.

The seismic grade MEMS accelerometers recently announced by HP [2-4] consist of a hermetically sealed stack of three silicon wafers. The first wafer has a cavity etched into the bulk silicon. The second wafer is plasma bonded to this cavity wafer, and receives a Bosch etch to release the proof mass and form the flexure springs on which the mass is suspended (Figure 1a,b). The third "cap" wafer is bonded to the above two-wafer assembly, providing sensing electronics and hermetically sealing the device.

The final etched dimensions of the flexure springs determine their stiffness and strongly impact the performance of the MEMS accelerometers. However, due to the depth and the high aspect ratio of the etched flexures, meaningful three-dimensional measurements are very difficult to obtain, while the behavior of the etch is more complex than can be characterized using top-down width measurements alone (Figure 1c). Although cross-section SEM micrographs were essential for our initial work, the timeintensive and destructive nature of the technique limited its usefulness for controlling manufacture of the accelerometers in commercial volumes.
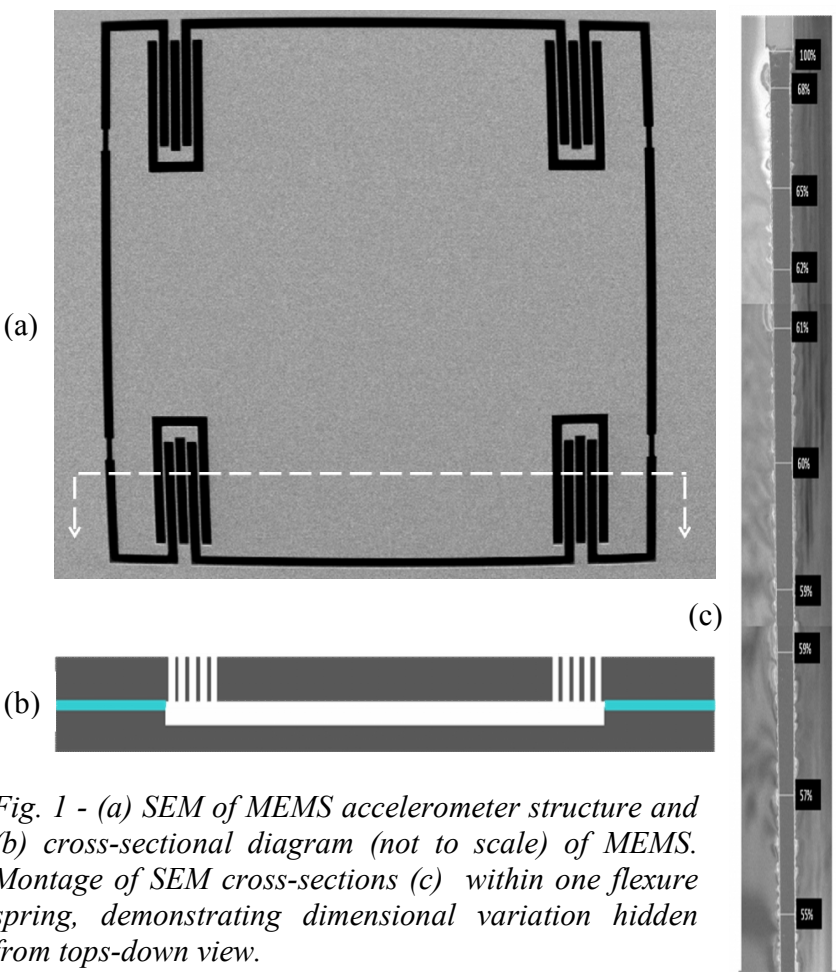

(b)

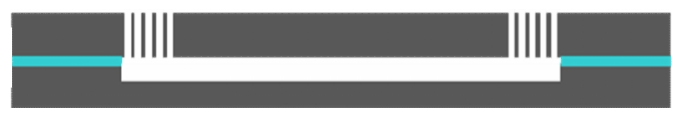

Fig. 1 - (a) SEM of MEMS accelerometer structure and (b) cross-sectional diagram (not to scale) of MEMS. Montage of SEM cross-sections (c) within one flexure spring, demonstrating dimensional variation hidden from tops-down view.

It was observed that the active MEMS elements will oscillate once etching has released the movable portion of the MEMS device (Figure 2). This movement can be recorded and analyzed to determine the resonant frequency of the MEMS structure, which is the critical parameter of interest. A simple technique to obtain this signal uses an intensity modulated fiber optic lever transducer.

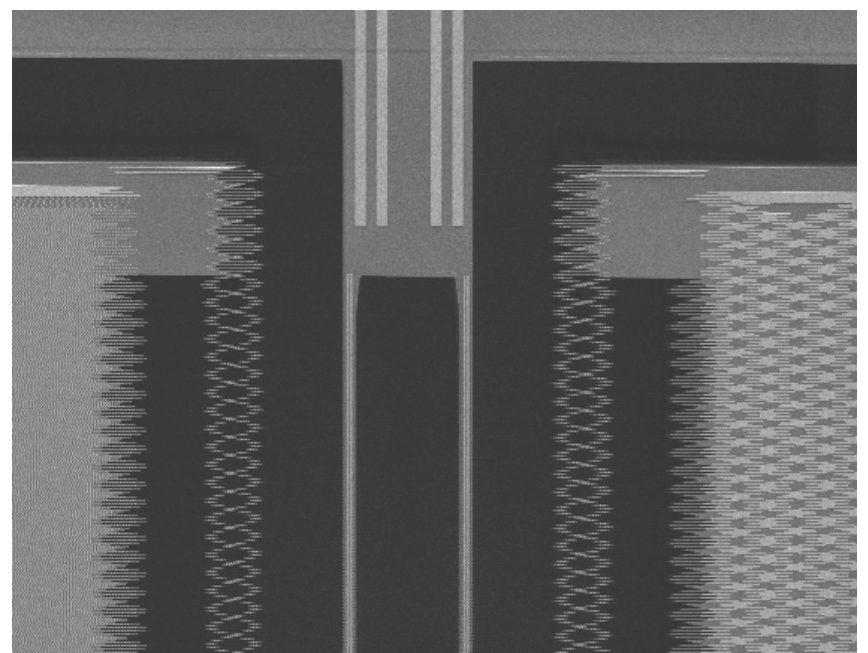

Figure 2-Observed oscillation of released accelerometer in SEM 
A fiber optic bundle in which adjacent fibers act as either illumination transmitting fibers or as reflected intensity receiving fibers will form an intensity-modulated optic lever displacement transducer [5], a transducer sensitive to displacement of a sample illuminated by the bundle. The intensity of the reflected light signal changes as a function of the distance to the illuminated sample, illustrated in Figures 3.
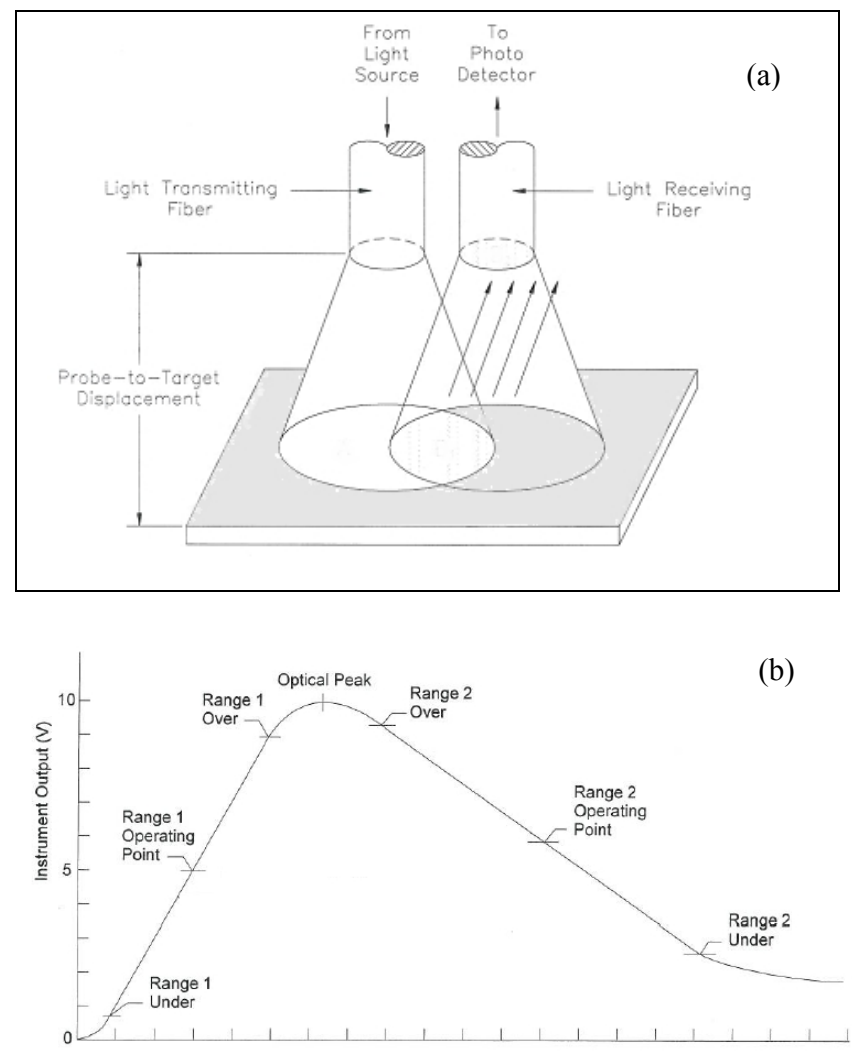

Probe to Target Gap, Displacement (uin or um)

Figure 3 - (a) Intensity modulated optic lever principle [6] and (b) Probe intensity calibration curve [6].

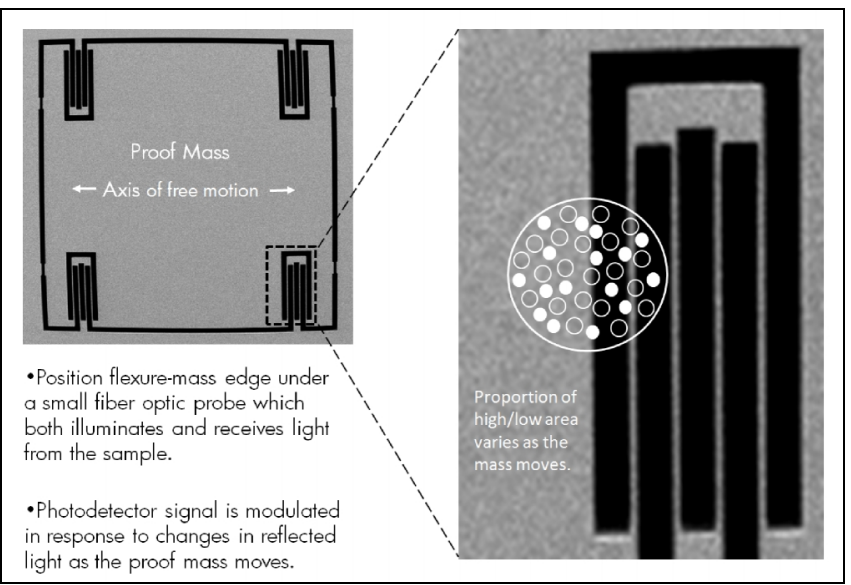

Figure 4 - Schematic of signal generation principal

By positioning the free moving portion of a MEMS device partially under a fiber optic transducer, the reflected signal produced is an area average of the signal from the unetched (high) and etched (low) portions of the device. By then stimulating its motion mechanically, the reflected light signal is modulated as the proportion of high and low topography under the beam changes (Figure 4). The signal data is fit to a damped sinusoidal model, from which the resonant frequency of the MEMS device, the initial amplitude and quality factor of the signal can be calculated.

\section{MANUAL SYSTEM \\ IMPLENTATION}

A manually operated measurement system was built, utilizing the fiber optic transducer principle. An MTI 2020R fiber optic probe was mounted to a microscope frame and wafer stage, allowing $\mathrm{X}$-, $\mathrm{Y}$ - and $\mathrm{Z}$-axis positioning of the individual die on sample wafers to be brought under the probe, shown in Figure 5. The optical probe is controlled by an MTI-2100 Fotonic Sensor, which converts the reflected light signal to a $0-10$ Volt electrical output signal and provides various options for filtering the output signal. The output voltage signal is routed to a DAQ board (Natl. Instr. PCIe-6321) in a PC, which samples and stores the signal. The stored signal can be analyzed with various techniques. In this case, the data are compared to a damped sinusoid model using a MATLAB program, and frequency, initial amplitude, quality factor and $\mathrm{R}^{2}$ are extracted from each set of fit data.

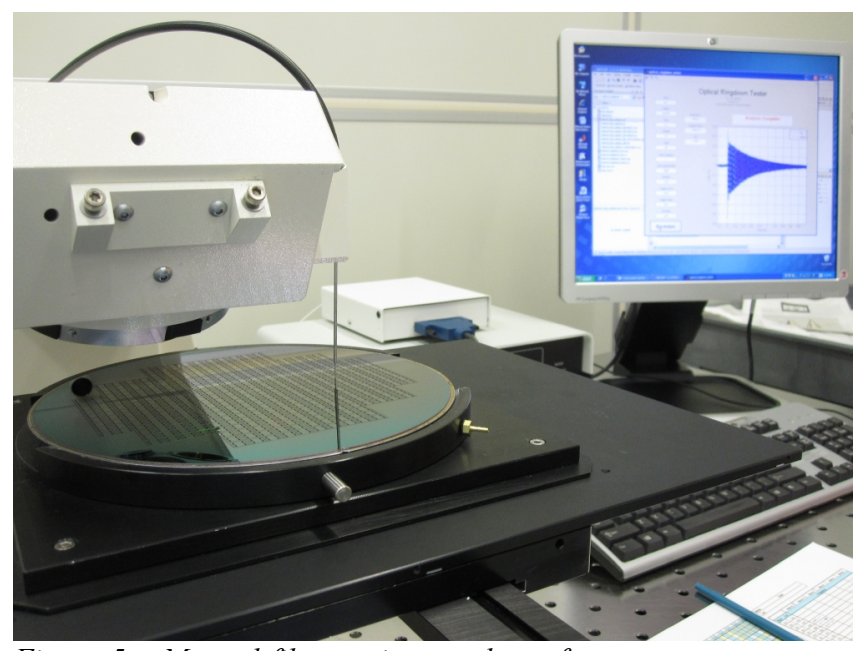

Figure 5 - Manual fiber optic transducer frequency measurement system

\section{OPERATION} follows:

Using the above apparatus, measurements are taken as

A sample wafer is loaded by hand onto the wafer stage, and the stage is moved to bring the unetched upper surface of the wafer assembly into the plane of focus of the optical probe. The probe is operated at the left (high) end of 'Range 2' as shown in Figure 3b, with the upper surface of the wafer assembly approximately $300 \mu \mathrm{m}$ from the probe tip. This returns a voltage signal of approximately 8 Volts with good contrast to the lower, etched regions, which provide a signal of roughly 2.5 Volts. The probe illumination intensity can be adjusted through the MTI-2100 Fotonic Sensor as needed.

Using the wafer stage the sample is moved, centering the edge of an etched flexure spring in the illuminated area under the fiber optic probe, causing the return signal voltage to fall to about half the value given by the unetched upper surface. The edge of the wafer assembly is then tapped gently with a metal rod to excite the accelerometers into motion, modulating the return signal, which is captured by the DAQ board and stored on the PC. The MATLAB program then analyzes the stored data, and logs the parameters to a 
text file. Using this manual system, the time to move, focus and measure each new sample on a wafer is about 30 seconds.

\section{RESULTS}

Due to the large volume of data and many cycles over which the signal can be obtained, measurements taken in this manner can be fit to the damped sinusoid model with correlation coefficients above 0.9 for typical measurements (Figure 6).

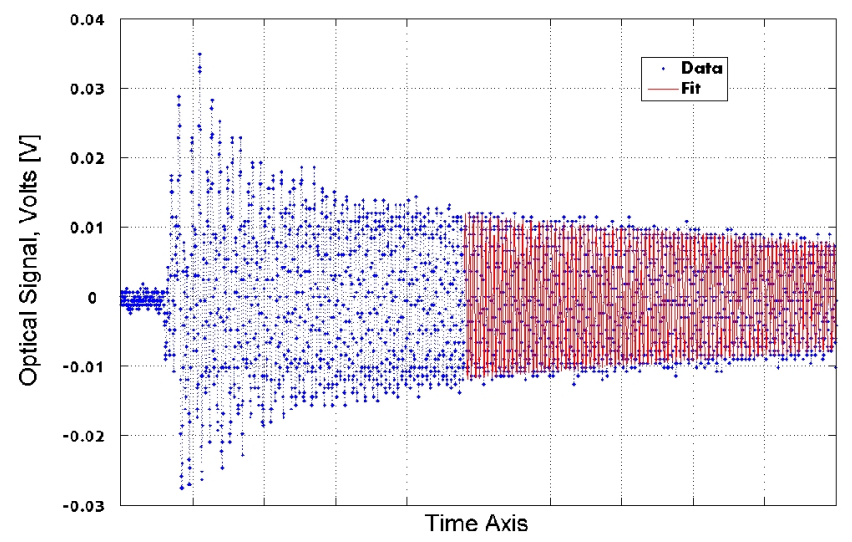

Figure 6-Typical optical signal fit to damped harmonic model.
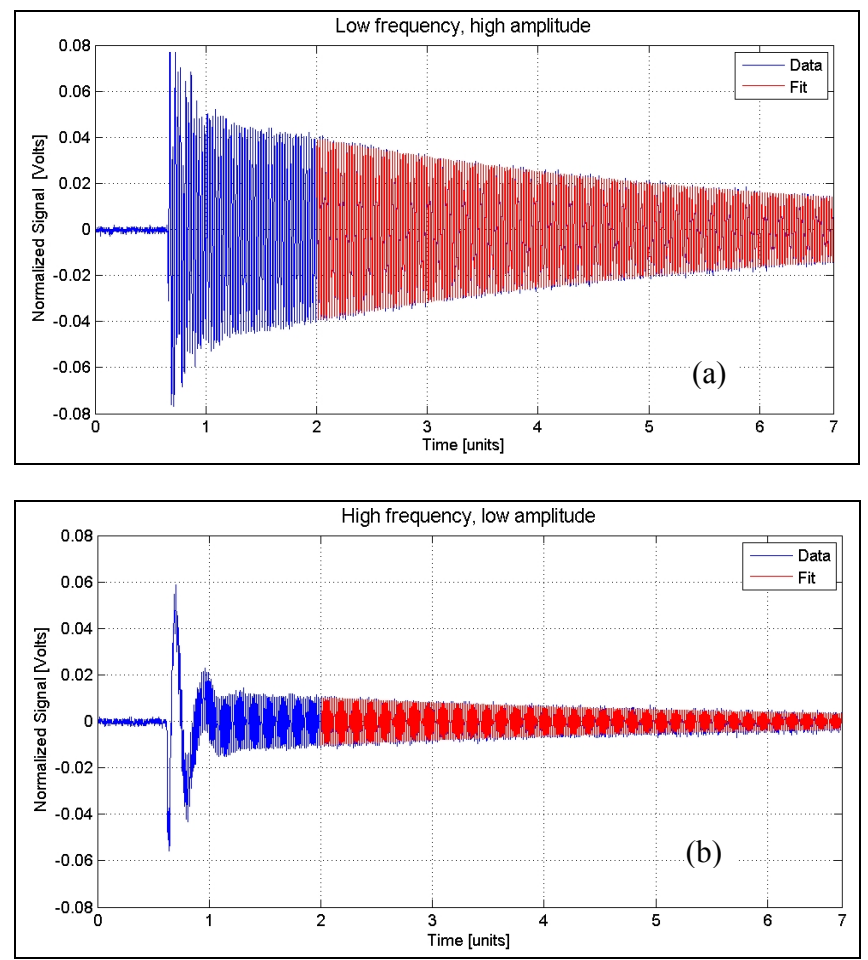

Figure 7 - Signals typical of (a) weak and (b) stiff flexure springs.

This data has proven extremely valuable when optimizing the BOSCH DRIE recipe parameters, making immediate feedback available as recipe parameter experiments were designed and etched. Over-etching and/or poor passivation were known to produce flexure springs with narrow cross-sectional shape, and low resonant frequencies and high amplitudes consistent with weak springs were measured for parts etched under those conditions (Figure 7a). Conversely, flexures which were under-etched or over-passivated during etch gave high resonant frequencies and lower amplitudes (Figure 7b) consistent with stiffer, wider springs. Improvements in across-the-wafer frequency response uniformity (Figure 8) were thus quickly obtained.

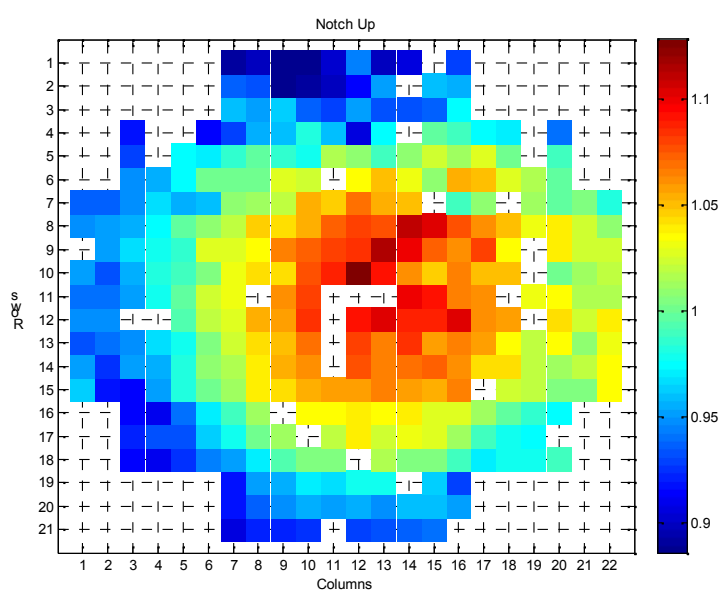

Figure 8-Typical normalized cross-wafer frequency uniformity.

The data obtained from this measurement system has also proven quite useful in diagnosing and resolving manufacturing process excursions. Whereas top-down width measurements failed to detect that a photolithography process shift had occurred, these optical measurements detected the shift in device frequency and allowed quick response. Additionally, brken or fractured flexure springs which are difficult to detect using automated top-down inspection techniques are readily identifiable within the frequency response data.

Most importantly, the optical data proved to be a reliable indicator of the frequency which can be expected from the finished devices when tested electrically, over a wide range of designs, frequencies and etch conditions (Figure 9).

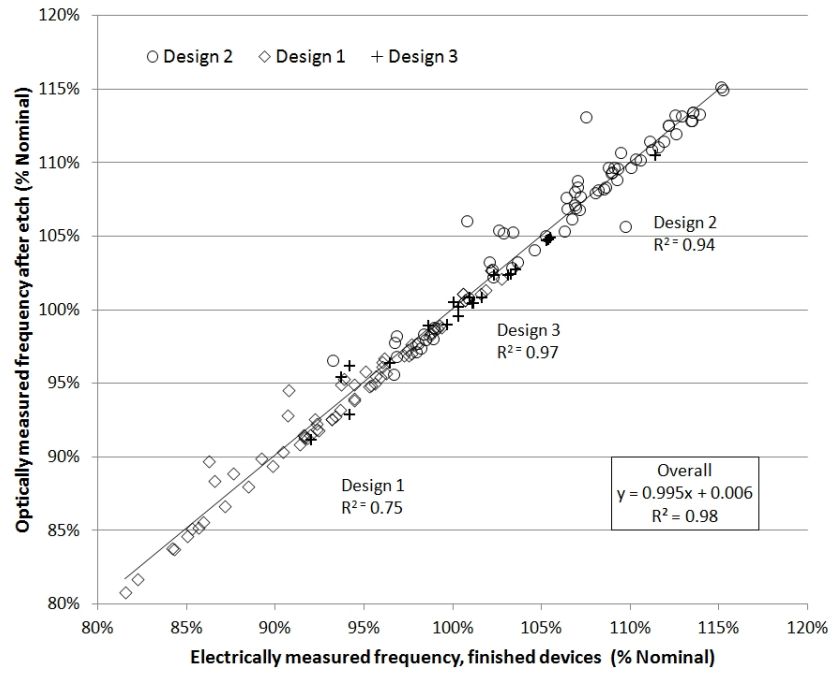

Figure 9 - Correlation of optical frequency measurements done in-process to electrical frequency measurements taken on finished devices. 


\section{AUTOMATED SYSTEM}

To provide the extensive data required to produce the HP seismic grade MEMS accelerometers in commercial volumes with demonstrated process control, a high degree of automation is required of the measurement system. As such, fiber optic transducers have been integrated into existing measurement equipment as an off-axis metrology system, allowing each device to be measured following release etch.

\section{IMPLENTATION}

An MTI 2020R fiber optic probe was fixed to each side of the microscope frame of an existing IVS-130, allowing access to the entire surface of the $200 \mathrm{~mm}$ wafer assemblies (Figure 10). To provide adequate working distance for wafer handling, each probe is fitted with a KD-LS-1A Fiber Optic Probe Extender (MTI), which increases the working distance to approximately $12 \mathrm{~mm}$. As on the manual system, the probes are controlled by an MTI-2100 Fotonic Sensor, converting the reflected light to a $0-10$ Volt electrical output signal and providing various options for filtering the output signal.

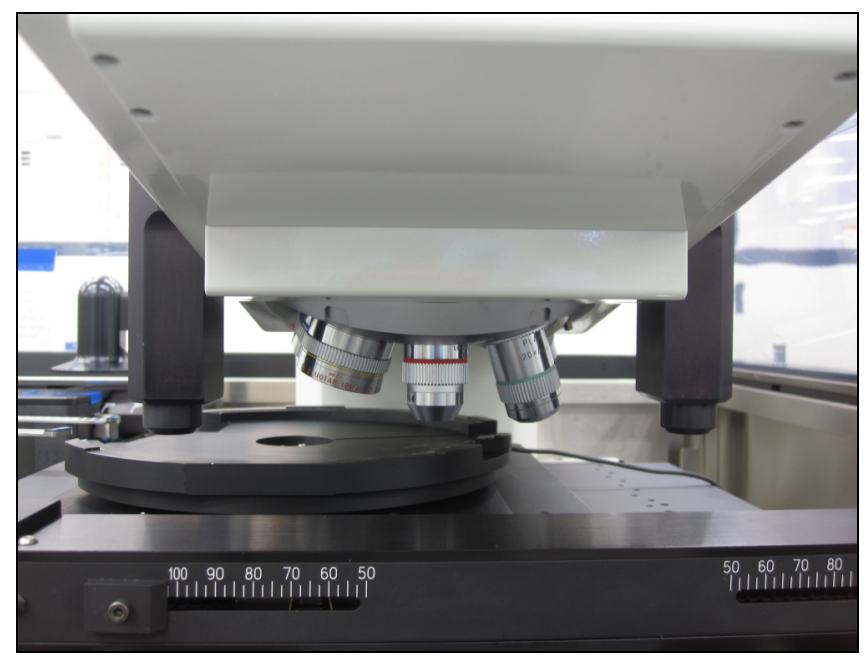

Figure 10: Fiber optic probes and extenders mounted to either side of IVS-130 microscope frame.

Following recipe selection via SECS and automated wafer loading, a modified control program on the IVS-130 routes the samples selected for measurement to either the left or right probe, based on the location of the sample on the wafer assembly. On the automated system, the output voltage signals are again routed to a DAQ board (Natl. Instr. PCIe-6321) housed in a separate PC, which samples, stores and analyzes the signal using proprietary software, and then provides the extracted measurement parameters to the computer controlling the IVS-130 (Figure 11). The IVS-130 control computer reports these parameters as it would any other dimension or overlay measurement.

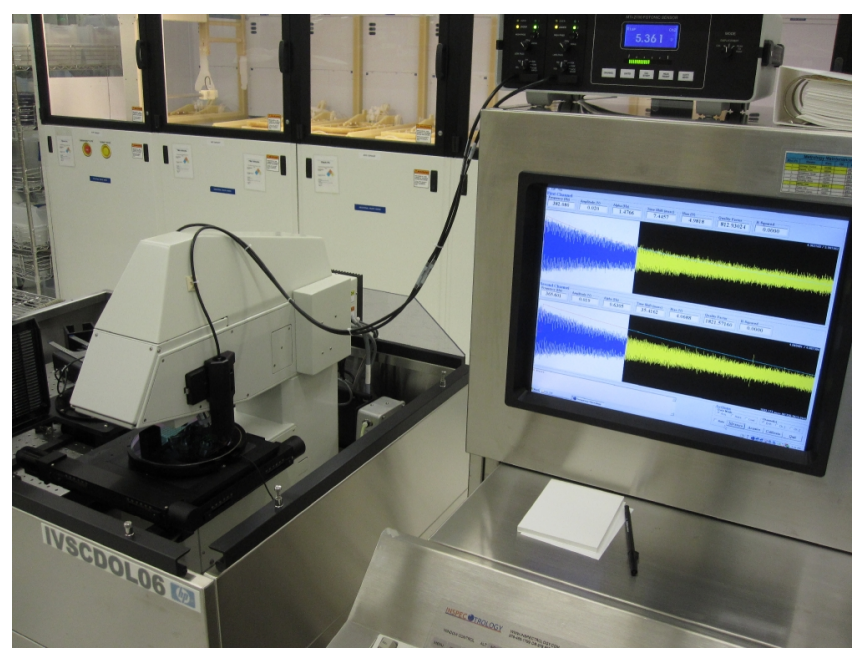

Figure 11: Automated system displaying signal analysis screen.

Rather than striking the samples, the automated system uses the motion of the wafer assembly on the stage to stimulate the accelerometers into motion. Driving the sample to a stop under the fiber optic probe results in a gentle acceleration to which the devices respond.

\section{RESULTS}

Although gentle, the wafer stage move and stop under the fiber optic probes provides sufficient stimulus to cause the accelerometers to resonate detectably (Figure 12). Again, due to the large volume of data and many cycles over which the signal is obtained, measurements taken in this manner can be fit to a damped sinusoid model with high confidence. Operating the optical probes in the manner described results in a sensitivity to the lateral motion of an edge of approximately $20 \mathrm{mV} / \mu \mathrm{m}$.

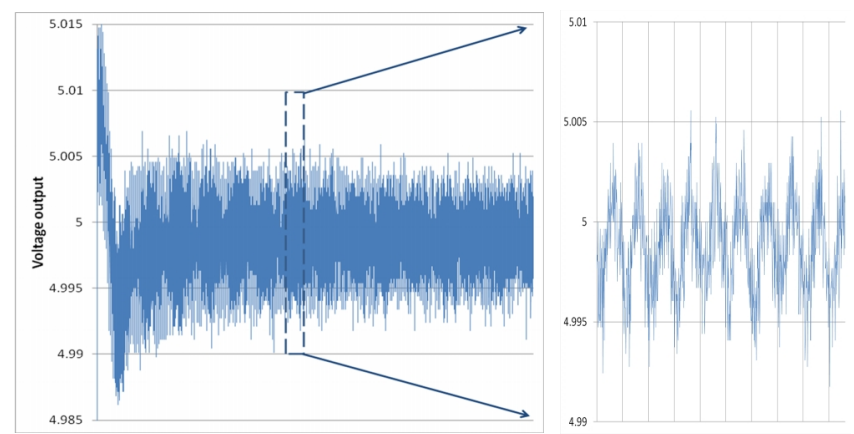

Figure 12: Initial 0.8secs of signal output (a) and section of same signal on an expanded time axis (b).

Data taken on the automated system matches data taken from the manual system to within a fraction of one Hertz in all instances (Figure 13). Both of the probes on the automated system are shown to match the single-probe manual system, thus they also match one another. 


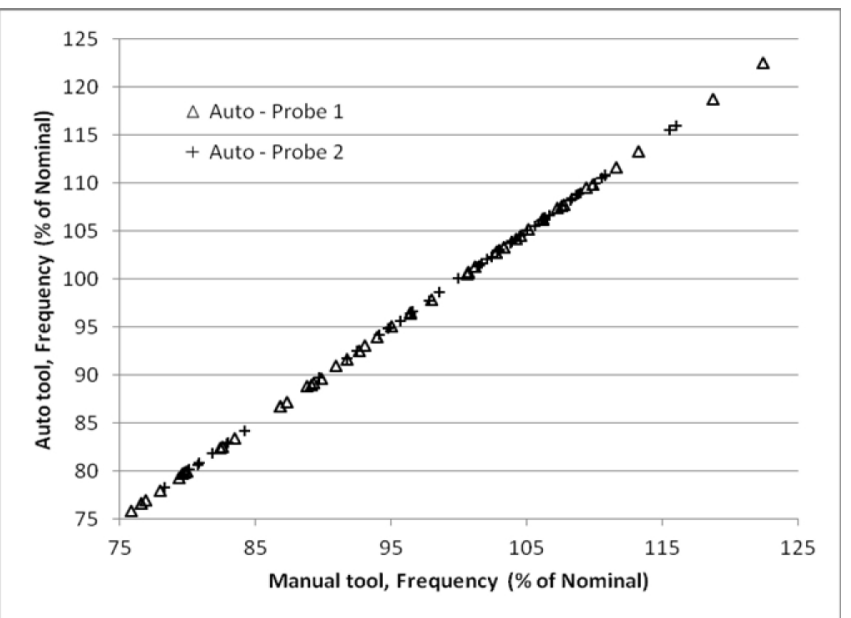

Figure 13: Correlation of frequency measurements taken on Automatic and Manual systems.

All of the original capabilities of the IVS measurement tool remain intact, and the tool still performs dimensional and overlay measurements. Frequency measurement data can be taken in the same program as, and interspersed with, dimensional or overlay data. For frequency measurement, move-acquire-measure (MAM) time is 11 seconds, allowing extensive datasets to be taken. The tool is SECS compliant and is interfaced to an automated factory control system for recipe selection and data transfer.

\section{CONCLUSION}

The resonant frequency of MEMS devices can be obtained by using a fiber optic transducer to detect the motion of the device. This relatively simple device - no lasers, no moving parts - was used to build a manual measurement system and has since been incorporated into an available automated tool as an off-axis measurement system, offering programmable sample selection, cassette-to-cassette wafer handling and recipe selection via SECS interface. Data obtained using this tooling has a high signal to noise ratio, is extremely repeatable, with excellent fit to the modeled damped sinusoidal behavior. These measurements can be conducted quickly and non-destructively immediately following the release etch and have proven quite useful in characterizing and controlling the fabrication processes used to form the flexure springs of the devices. The measurements also correlate very well to the resonant frequency as determined electrically in the completed devices.

\section{ACKNOWLEDGEMENT}

The authors would like to thank Mr. Paul Slazas of MTI for his advice and assistance in selecting and using the fiber optics and instruments.

\section{REFERENCES}

[1] F. Laermer and A. Urban, "BOSCH-DRIE Shaping MEMS History, Applications and Future Directions", Technical Digest of the 2010 Solid-State Sensors, Actuators, and Microsystems Workshop, Hilton Head Isl.,SC 6/6-10/10, Transducer Research Foundation, Cleveland, OH (2010), pp. 1-6.

[2] HP News Release (2010, Feb. 15), "Shell and HP to Develop Ultrahigh-Resolution Seismic Sensing Solution” [Online]. Available: http://www.hp.com/hpinfo/newsroom/press/2010/100215xa.ht $\underline{\mathrm{ml}}$

[3] B.D. Homeijer, et.al., "Hewlett-Packard's Seismic Grade MEMS Accelerometer", Proc.24th IEEE Conf. on MEMS, Cancun,MX, Jan. 2011

[4] D.J. Milligan, B.D. Homeijer and R.G. Walmsley, "An UltraLow Noise MEMS Accelerometer for Seismic Imaging", Proc.10th IEEE Conf. on Sensors, Limerick,IE, Nov. 2011

[5] R.O. Cook and C.W. Hamm, "Fiber optic lever displacement transducer”, Applied Optics, Vol.18, No. 19, pp. 3230-3241, Oct. 1, 1979.

[6] MTI Instruments, "An Introduction to the Fotonic Sensor", Application notes [On-line] Available: http://www.mtiinstruments.com/products/fiberopticmeasurem ent.aspx

\section{CONTACT}

*B. Keese, tel: +1-541-715-2497; william.keese@hp.com 